Check for updates

Cite this: Mater. Adv., 2022, 3, 998

Received 9th August 2021 Accepted 17th November 2021

DOI: $10.1039 / \mathrm{d} 1 \mathrm{ma} 00704 a$

rsc.li/materials-advances

\title{
Synthesis of polar polynorbornenes with high dielectric relaxation strength as candidate materials for dielectric applications $\dagger$
}

\author{
Francis Owusu, (DD ${ }^{\text {ab }}$ Martin Tress, ${ }^{c}$ Frank A. Nüesch, iD ${ }^{\text {abd }}$ Sandro Lehner ${ }^{\mathrm{e}}$ and \\ Dorina M. Opris (iD *a
}

\begin{abstract}
Materials with high dielectric permittivity and dielectric relaxation strength are sought for thermal and pressure sensors and electrical energy generators. However, most polymers have either too low dielectric permittivity or are so polar that their glass transition temperature $\left(T_{\mathrm{g}}\right)$ is too high and thus decomposition and side reactions occur before an electric field can polarize the polar groups. Here, we use the power and versatility of ring-opening metathesis polymerization (ROMP) to synthesize polar polymers with high dielectric relaxation strength and $T_{\mathrm{g}}$ significantly below the decomposition temperature. We first synthesized six polar norbornene monomers by conventional esterification, which were then polymerized by ROMP using Grubbs first- and third-generation catalysts. The structure of the polynorbornenes obtained were verified by multinuclear NMR spectroscopy, molecular weights determined by gel permeation chromatography (GPC), and thermal properties evaluated by thermogravimetric analysis (TGA) and differential scanning calorimetry (DSC). Additionally, their dielectric permittivity, conductivity, and dielectric losses were measured at different temperatures and frequencies ranging between 0.1 and $10^{6} \mathrm{~Hz}$.
\end{abstract}

\section{Introduction}

The design and synthesis of polymers responsive to different stimuli is a research field that gained significant attention from polymer chemists. ${ }^{1}$ A large body of research has been focused on achieving polymeric materials that respond to $\mathrm{pH}$, humidity, temperature, light, and magnetic field. However, little attention has been paid to the synthesis of electroactive polymers despite their potential in various applications. ${ }^{2}$

Electret polymers have a quasi-permanent polarization and respond to mechanical or thermal stress by generating an electric signal, and thus show piezo and pyroelectric effects. ${ }^{3}$

\footnotetext{
${ }^{a}$ Swiss Federal Laboratories for Materials Science and Technology Empa, Laboratory for Functional Polymers, Überlandstr. 129, CH-8600, Dübendorf, Switzerland. E-mail: dorina.opris@empa.ch

${ }^{b}$ Institute of Chemical Sciences and Engineering, Ecole Polytechnique Federale de Lausanne, EPFL, Station 6, CH-1015 Lausanne, Switzerland

${ }^{c}$ Leipzig University, Peter Debye Institute for Soft Matter Physics, Linné straße 5, 04103 Leipzig, Germany

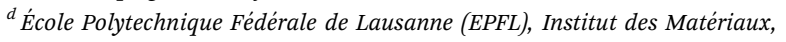
Station 12, CH 1015, Lausanne, Switzerland

${ }^{e}$ Swiss Federal Laboratories for Materials Science and Technology Empa,

Laboratory for Advanced Fibers, Lerchenfeldstrasse 5, 9014 St. Gallen, Switzerland $\dagger$ Electronic supplementary information (ESI) available: ${ }^{1} \mathrm{H}$ and ${ }^{13} \mathrm{C}$ NMR spectra, MS, elemental analysis, DSC, TGA, impedance spectroscopy data. See DOI: 10.1039/d1ma00704a
}

These effects were known, but a significant advancement in this field was achieved only when the piezoelectric response in polyvinylidene difluoride (PVDF) was discovered in $1969 .{ }^{4}$ Since then, many applications for this polymer were found, which range from sensors, actuators, energy conversion to electronic devices. ${ }^{5,6}$ However, PVDF is environmentally unfriendly and difficult to process. A further advancement in the field was achieved when small air voids in highly insulating polymer foams were polarized by corona discharge. ${ }^{7}$ Such foams show a high piezoelectric response, but humidity negatively influences their performance. ${ }^{8}$

Quasi-permanent polarization can be introduced in amorphous polar polymers by poling in a strong electric field when heated above $T_{\mathrm{g}}$ and cooled below $T_{\mathrm{g}}{ }^{9}$ Research in this direction attracted the attention of polymer chemists in the 1990s, as for example Hall Jr., ${ }^{10}$ Feast, ${ }^{11}$ and Wegner. ${ }^{12}$ Polyacrylonitrile and poly(vinylidenecyanide vinyl acetate) (PVVA) show a large dielectric relaxation strength $(\Delta \varepsilon)$, but they are either environmentally unfriendly or have a high $T_{\mathrm{m}}$. PVVA has amongst the highest $\Delta \varepsilon=30 .{ }^{13}$ While this is attractive, its very high $T_{\mathrm{g}}$ $\left(170{ }^{\circ} \mathrm{C}\right)$ complicates the poling process due to possible thermal decomposition and side reactions. To achieve a practical piezoelectric response, polymers with as high as possible $\Delta \varepsilon$ are desirable. The dielectric relaxation strength is defined as the difference between the $\varepsilon_{\mathrm{r}}$ and $\varepsilon_{\infty}$ and is related to the density of 
polar groups $(N)$ and the dipole moment $(\mu)$. The polarization $(P)$ is given by

$$
P=\varepsilon_{0} \Delta \varepsilon(T) E_{\mathrm{p}}
$$

where $\varepsilon_{0}$ is the vacuum permittivity, $\Delta \varepsilon(T)$ the dielectric relaxation strength at the poling temperature, and $E_{\mathrm{p}}$ the poling field.

Polymers with large $\Delta \varepsilon$ can be accessed by increasing the dipole density and dipole strength of polar groups grafted to the polymer chain. Polar groups can be introduced into a polymer chain either by post-polymerization modification or by polymerizing polar monomers. Ring-opening polymerization (ROMP) is a powerful tool for synthesizing polymers with different functional groups. ${ }^{14,15}$ Norbornene-based monomers are predominantly employed due to their high ring strain, ensuring excellent polymerization propagation. Additionally, this monomer class can easily be modified with a wide range of functional groups, giving rise to polymers with otherwise inaccessible structures and properties. ${ }^{16,17}$ Due to this versatility, polynorbornenes with pendant groups in the polymer backbone have recently spotlighted materials research. This is also due to the materials' attractive optical and electro-optic properties, ${ }^{18}$ good mechanical and adhesive properties, ${ }^{19-21}$ multi-shape memory effects, ${ }^{22}$ and excellent dielectric behavior. ${ }^{23-28}$ To increase the dielectric permittivity, functional moieties with large dipole moments as side chains have typically been considered. ${ }^{29}$ For instance, Feast reported the synthesis of poly[2,3-bis(trifluoromethyl)norbornadiene] and its dielectric and pyroelectric properties. ${ }^{11}$ Most recently, Bonardd et al. reported on the synthesis of new poly(itaconate) polymers containing pendant sulfone and nitrile groups, which showed an interesting dielectric behavior in a broad temperature range, proving their potential as promising candidates for energy storage applications. ${ }^{30,31}$

There are only a few systematic experimental studies available on how different dipole moieties as pendant groups of poly norbornenes affect dielectric properties. Here, we report the synthesis of six polar norbornene monomers and their ROM polymerization. Furthermore, we report the thermal and dielectric properties of these polynorbornenes. Gratifyingly, some of these polymers show high $\Delta \varepsilon$ and a $T_{\mathrm{g}}$ suitable for room temperature applications.

\section{Experimental}

\section{Materials}

All reagents were purchased from Sigma Aldrich and utilized without further purification unless otherwise stated. 2,6-Dimethyl$4 H$-pyran-4-one and 1-ethyl-3-(3-dimethylaminopropyl) carbodiimide (EDC) were acquired from ABCR GmbH and TCI Chemicals, respectively. Ethyl acetate, ethanol, methanol, heptane, dichloromethane (DCM), toluene, and tetrahydrofuran (THF) were purchased from VWR Chemicals and deuterated solvents from Deutero GmbH. N-Methyl-4-nitroaniline was synthesized according to the literature. ${ }^{32}$ DCM was dried over calcium hydride. Millipore Milli-Q system processed deionized water was taken for all experimental work-ups.

\section{Standard measurements and instrumentation}

${ }^{1} \mathrm{H}$ and ${ }^{13} \mathrm{C}$ NMR spectra were recorded on a Bruker Avance-400 spectrometer (400 $\mathrm{MHz}$ at room temperature) using $\mathrm{CDCl}_{3}$ as a solvent if not otherwise stated. Mass spectra of monomers were obtained using a Bruker Daltonics HPLC-ESI-qTOF-MS spectrometer. Elemental analyses were conducted on a LECO TruSpec Micro and a LECO 628 O Micro employing IR spectroscopy as a detection unit.

The average molecular weights and polydispersity indices (PDI) of polymers were determined by two different gel permeation chromatography (GPC) systems with THF or hexafluoroisopropanol (HFIP) as eluents. The THF-based setup was equipped with Agilent 1260 Infinity, using two tandem-connected mixed-bed columns $(1 \times$ PLgel $5 \mu \mathrm{m}$ MIXED-C Guard and $2 \times$ PLgel $5 \mu \mathrm{m}$ MIXED-C Analytical), coupled to a 390-MDS refractive index detector. Both systems employed a flow rate of $1 \mathrm{ml} \mathrm{min}^{-1}$ and were kept at $35-40{ }^{\circ} \mathrm{C}$. Polystyrene and poly(methyl methacrylate) were used as a calibration standard for the THF and HFIP eluent systems, respectively.

The thermal behavior of the synthesized polymers was investigated using a PerkinElmer DSC 8000 differential scanning calorimeter. The samples were initially heated to $150{ }^{\circ} \mathrm{C}$ at a rate of $20{ }^{\circ} \mathrm{C} \min ^{-1}$ to remove any thermal history. Heat-cool-heat cycles were scanned from 0 to $200{ }^{\circ} \mathrm{C}$ at a rate of $20{ }^{\circ} \mathrm{C} \mathrm{min}^{-1}$. For samples PNBE-3 a heating-cooling-heating cycles from 0 to $150{ }^{\circ} \mathrm{C}$ at the rate of $20{ }^{\circ} \mathrm{C} \mathrm{min}{ }^{-1}$ were used. Thermogravimetric analysis (TGA) was conducted using a PerkinElmer TGA7 at a heating rate of $10{ }^{\circ} \mathrm{C} \mathrm{min}^{-1}$ under a nitrogen gas flow.

Broadband dielectric spectroscopy (BDS) measurements were performed using a Novocontrol Alpha-A Frequency Analyzer equipped with quatro cryosystem temperature control. Samples were prepared by making pellets of the polymers with the aid of a hydraulic press, sandwiching between two metal electrodes with $100 \mu \mathrm{m}$ glass fibres as spacers, and melt pressing in the $110{ }^{\circ} \mathrm{C}$ to $130{ }^{\circ} \mathrm{C}$ temperature range. Dielectric spectra were obtained by applying an external electric field of $10 \mathrm{kV} \mathrm{m}^{-1}$ in a frequency and temperature range of 0.1 to $1 \mathrm{MHz}$ and -100 to $160{ }^{\circ} \mathrm{C}$, respectively.

\section{Synthetic methods}

Synthesis of 2-(1-(2-hydroxyethyl)-2,6-dimethylpyridin-4(1H)ylidene)malononitrile. 2,6-Dimethyl- $4 H$-pyran-4-one $(20.00 \mathrm{~g}$, $161.1 \mathrm{mmol})$ and malononitrile $(10.64 \mathrm{~g}, 161.1 \mathrm{mmol})$ were dissolved in acetic anhydride $(80 \mathrm{ml})$ and the solution charged into a $200 \mathrm{ml}$ round bottom flask. The reaction mixture was refluxed at $130{ }^{\circ} \mathrm{C}$ for $4 \mathrm{~h}$ to obtain crude $\mathbf{4 H}$-PMal intermediate (Scheme 1). The intermediate was purified by washing the unreacted acetic acid with warm water and recrystallizing from heptane to produce a dark brown powder ( $87 \%$ yield).

A $200 \mathrm{ml}$ round bottom flask was charged with $4 \mathrm{H}$-PMal intermediate $(15.00 \mathrm{~g}, 87 \mathrm{mmol})$, ethanolamine $(44.7 \mathrm{ml}$, $745 \mathrm{mmol})$ and methanol $(100 \mathrm{ml})$. The reaction was refluxed 


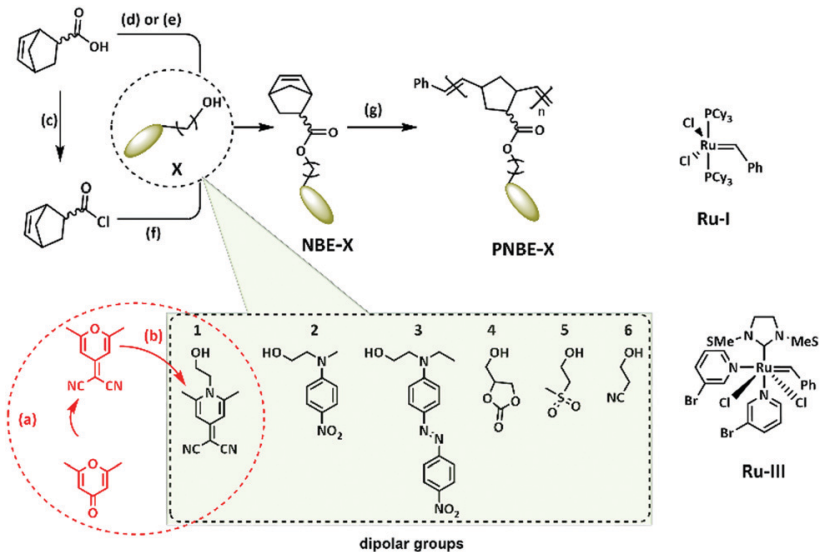

Scheme 1 Synthesis of monomers and respective homopolymers: (a) malononitrile, acetic anhydride, $4 \mathrm{~h}$, reflux; (b) ethanolamine, methanol, 2 h, rf; (c) thionyl chloride, anhydrous chloroform 4 h, rf; (d) 4dimethylaminopyridine, $N, N^{\prime}$-dicyclohexylcarbodiimide, dry DCM, $24 \mathrm{~h}$, $45{ }^{\circ} \mathrm{C}$; (f) $\mathrm{N}, \mathrm{N}$-dimethylaniline, anhydrous chloroform, $13 \mathrm{~h}, 0{ }^{\circ} \mathrm{C}$ to rf; (g) Grubbs 1st or 3rd generation catalysts, dry DCM, $18 \mathrm{~h}, 40{ }^{\circ} \mathrm{C}$.

at $70{ }^{\circ} \mathrm{C}$ for $2 \mathrm{~h}$ and then it was left to stand overnight. The separated solid was collected by filtration, dried, and recrystallized in ethanol to give compound $\mathbf{1}$ as brown flakes (yield $60 \%$ ). ${ }^{1} \mathrm{H}$ NMR (DMSO- $\left.d_{6}\right) \delta 6.68(\mathrm{~s}, 2 \mathrm{H}, \mathrm{Ar}-\mathrm{H}), 5.17(\mathrm{t}, J=$ $5.4 \mathrm{~Hz}, 1 \mathrm{H}, \mathrm{OH}), 4.17\left(\mathrm{t}, J=5.6 \mathrm{~Hz}, 2 \mathrm{H}, \mathrm{N}-\mathrm{CH}_{2}\right), 3.70(\mathrm{q}, J=$ $5.4 \mathrm{~Hz}, 2 \mathrm{H}, \mathrm{CH}_{2}-\mathrm{OH}$ ), 2.53 (s, $\left.6 \mathrm{H}, \mathrm{Ar}-\mathrm{CH}_{3}\right) ;{ }^{13} \mathrm{C}$ NMR $\left(\mathrm{DMSO}-d_{6}\right)$ $\delta 155.40\left(\mathbf{C}_{\mathbf{A r}}=\mathrm{C}(\mathrm{CN})_{2}\right), 150.92\left(\mathbf{C}_{\mathbf{A r}}-\mathrm{CH}_{3}\right), 119.43(\mathbf{C N}), 113.07$ $\left(\mathbf{C}_{\mathbf{A r}}-\mathrm{H}\right), 59.76\left(=\mathbf{C}(\mathrm{CN})_{2}\right.$ and $\left.\mathrm{CH}_{2}-\mathrm{OH}\right), 51.13\left(\mathrm{~N}-\mathrm{CH}_{2}\right), 21.03$ $\left(\mathrm{Ar}-\mathrm{CH}_{3}\right.$ ); $\mathrm{MS}$ (ESI) $\mathrm{m} / z$ calcd for $\mathrm{C}_{12} \mathrm{H}_{13} \mathrm{~N}_{3} \mathrm{NaO}[\mathrm{M}+\mathrm{Na}]^{+}$: 238.0951; found: 238.0950; Anal. Calcd for $\mathrm{C}_{12} \mathrm{H}_{13} \mathrm{~N}_{3} \mathrm{O}$ (\%): $\mathrm{C} 66.96, \mathrm{H} 6.09, \mathrm{~N}$ 19.52, O 7.43; found: $\mathrm{C} 66.91, \mathrm{H} 6.01$, N 19.43, O 7.31. (Fig. S1-S6, ESI $\dagger$ ).

\section{General preparation procedure for monomers (NBE-1 to NBE-4) via Steglich esterification}

A dried 2-necked round bottom flask was charged with 5-norbornene-2-carboxylic acid (1 equiv.), functional alcohol 1-6 ( 1 equiv.), and 4-dimethylaminopyridine ( 2 equiv.) dissolved in anhydrous dichloromethane $(60 \mathrm{ml}) . \quad N, N^{\prime}$-Dicyclohexylcarbodiimide (DCC)/1-ethyl-3-(3-dimethylaminopropyl)carbodiimide (EDC) (2 equiv.) was added at $0{ }^{\circ} \mathrm{C}$ to the reaction mixture, which was then stirred for $5 \mathrm{~min}$ at $0{ }^{\circ} \mathrm{C}$ and refluxed for $24 \mathrm{~h}$ at $45^{\circ} \mathrm{C}$. The resulting crude mass was concentrated in a vacuum and purified via column chromatography eluting with heptane and ethyl acetate $(3: 2)$. (Fig. S7-S34, ESI $\dagger$ ).

NBE-1 was obtained as a light yellow powder (59\% yield): ${ }^{1} \mathrm{H}$ NMR $\delta: 6.65$ (s, 2H), 6.19 (ddd, $\left.J=12.9,5.7,3.0 \mathrm{~Hz}, 1 \mathrm{H}\right), 5.96$ (ddd, $J=117.3,5.7,3.0 \mathrm{~Hz}, 1 \mathrm{H}), 4.41-4.18(\mathrm{~m}, 4 \mathrm{H}), 3.22-3.07$ (m, 1H), 3.01-2.87 (m, 2H), $2.53(\mathrm{~s}, 6 \mathrm{H}), 1.94(\mathrm{ddd}, J=11.8,9.4$, $3.7 \mathrm{~Hz}, 1 \mathrm{H}), 1.52-1.25$ (m, 3H). ${ }^{13} \mathrm{C}$ NMR (101 MHz, Chloroform- $d$ ) $\delta 175.78,174.23,156.07,147.86,147.84,138.42,138.31$, $135.38,131.80,118.36,113.85,113.83,61.17,61.07,49.74$, 46.83, 46.74, 46.53, 46.36, 45.72, 43.17, 42.85, 42.48, 41.59, $30.59,29.41,21.01,20.99$. MS (ESI) $m / z$ calcd for $\mathrm{C}_{20} \mathrm{H}_{21} \mathrm{~N}_{3} \mathrm{NaO}_{2}$ $[\mathrm{M}+\mathrm{Na}]^{+}:$358.1526; found: 358.1529. Anal. Calcd for
$\mathrm{C}_{20} \mathrm{H}_{21} \mathrm{~N}_{3} \mathrm{O}_{2}$ (\%): C 71.62, $\mathrm{H}$ 6.31, N 12.53, O 9.54; found: $\mathrm{C}$ 71.47, H 6.37, N 12.53 O 9.26. Refractive index: 1.565; density: 1.238 .

NBE-2 was obtained as a viscous yellow liquid (92\% yield): ${ }^{1} \mathrm{H}$ NMR $\delta 8.18-8.10(\mathrm{~m}, 2 \mathrm{H}), 6.74-6.66(\mathrm{~m}, 2 \mathrm{H}), 6.16(\mathrm{ddd}, J=$ $14.8,5.7,3.0 \mathrm{~Hz}, 1 \mathrm{H}), 6.10-5.81(\mathrm{~m}, 1 \mathrm{H}), 4.36-4.19(\mathrm{~m}, 2 \mathrm{H})$, $3.78-3.65(\mathrm{~m}, 2 \mathrm{H}), 3.14(\mathrm{~d}, J=2.2 \mathrm{~Hz}, 4 \mathrm{H}), 2.97-2.86(\mathrm{~m}, 2 \mathrm{H})$, $1.94-1.80(\mathrm{~m}, 1 \mathrm{H}), 1.48-1.40(\mathrm{~m}, 1 \mathrm{H}), 1.41-1.23(\mathrm{~m}, 2 \mathrm{H})$. ${ }^{13} \mathrm{C}$ NMR $\left(101 \mathrm{MHz}, \mathrm{CDCl}_{3}\right) \delta 176.14,174.66,153.43,138.16$, 138.07, 137.43, 135.54, 132.09, 126.18, 126.16, 110.53, 110.50, $60.98,60.82,50.90,50.85,49.68,46.48,46.36,45.65,43.28$, 43.05, 42.50, 41.58, 38.97, 38.96, 30.37, 29.27. MS (ESI) $\mathrm{m} / \mathrm{z}$ calcd for $\mathrm{C}_{17} \mathrm{H}_{20} \mathrm{~N}_{2} \mathrm{NaO}_{4}[\mathrm{M}+\mathrm{Na}]^{+}$: 339.1315; found: 339.1315 . Anal. Calcd for $\mathrm{C}_{17} \mathrm{H}_{20} \mathrm{~N}_{2} \mathrm{O}_{4}$ (\%): C 64.54, H 6.37, N 8.86, O 20.23; found: $\mathrm{C} 64.68, \mathrm{H} 6.54, \mathrm{~N}$ 8.92. Refractive index: 1.582; density: 1.237 .

NBE-3 was obtained as a reddish powder (95\% yield): ${ }^{1} \mathrm{H}$ NMR $\delta$ 8.43-8.24 (m, 2H), 8.04-7.82 (m, 4H), 6.90-6.74 $(\mathrm{m}, 2 \mathrm{H}), 6.24-6.13(\mathrm{~m}, 1 \mathrm{H}), 6.01(\mathrm{ddd}, J=77.5,5.7,3.0 \mathrm{~Hz}$, $1 \mathrm{H}), 4.30(\mathrm{dt}, J=26.8,6.3 \mathrm{~Hz}, 2 \mathrm{H}), 3.70(\mathrm{dt}, J=15.8,6.3 \mathrm{~Hz}, 2 \mathrm{H})$, $3.56(\mathrm{q}, J=7.2 \mathrm{~Hz}, 2 \mathrm{H}), 3.24-3.10(\mathrm{~m}, 1 \mathrm{H}), 3.05-2.87(\mathrm{~m}, 2 \mathrm{H})$, $2.02-1.89(\mathrm{~m}, 2 \mathrm{H}), 1.44(\mathrm{ddd}, J=11.1,7.6,4.1 \mathrm{~Hz}, 2 \mathrm{H}), 1.28(\mathrm{t}, J=$ $7.1 \mathrm{~Hz}, 3 \mathrm{H}) .{ }^{13} \mathrm{C}$ NMR $\left(101 \mathrm{MHz}, \mathrm{CDCl}_{3}\right) \delta 175.69(\mathrm{~d}, J=$ $106.1 \mathrm{~Hz}), 174.71,156.76,151.32,147.36,143.82,138.14$, 138.02, 136.86, 135.63, 132.53, 132.19, 126.27, 124.66, 122.64, $111.47,61.32,61.18,55.94,55.74,49.81,49.76,49.70,48.85$, $48.79,46.81,46.57,46.39,45.71,45.65,45.61,44.36,43.31$, $43.08,42.75,42.54,41.63,34.94,33.97,32.87,32.80,31.27$, $31.14,30.85,30.44,29.29,26.48,26.41,25.53,25.47,25.40$, 24.99, 24.86, 24.70, 12.30. MS (ESI) $\mathrm{m} / z$ calcd for $\mathrm{C}_{24} \mathrm{H}_{26} \mathrm{~N}_{4} \mathrm{NaO}_{4}$ $[\mathrm{M}+\mathrm{Na}]^{+}:$457.1846; found: 457.1848. Anal. Calcd. for $\mathrm{C}_{24} \mathrm{H}_{26} \mathrm{~N}_{4} \mathrm{O}_{4}$ (\%): C 66.34, H 6.03, N 12.89, O 14.73; found: C 67.97, H 6.94, N 12.13. Refractive index: 1.422; density: 1.185.

NBE-4 was obtained as a viscous, slightly yellow liquid $(90 \%$ yield): ${ }^{1} \mathrm{H}$ NMR $\delta$ 6.28-6.15 (m, 1H), 6.14-5.88 (m, 1H), 5.02$4.84(\mathrm{~m}, 1 \mathrm{H}), 4.57(\mathrm{td}, J=8.6,2.8 \mathrm{~Hz}, 1 \mathrm{H}), 4.44-4.16(\mathrm{~m}, 3 \mathrm{H})$, $3.32-3.18(\mathrm{~m}, 1 \mathrm{H}), 3.09-2.99(\mathrm{~m}, 1 \mathrm{H}), 2.95(\mathrm{dp}, J=5.3,2.2 \mathrm{~Hz}$, $1 \mathrm{H}), 2.01-1.88(\mathrm{~m}, 1 \mathrm{H}), 1.45$ (ddddd, $J=18.6,9.5,6.8,5.2$, $2.1 \mathrm{~Hz}, 2 \mathrm{H}), 1.30(\mathrm{dq}, J=8.3,1.7 \mathrm{~Hz}, 1 \mathrm{H}) .{ }^{13} \mathrm{C}$ NMR $\delta 175.79$, $174.25,154.47,138.34,138.20,138.15,135.51,132.14,131.96$, $73.92,73.90,73.89,73.84,66.02,65.99,62.98,62.87,49.79$, 49.72, 46.66, 46.63, 46.37, 45.84, 43.20, 43.18, 42.93, 42.51, $42.50,41.65,30.47,29.23$. MS (ESI) $\mathrm{m} / z$ calcd for $\mathrm{C}_{12} \mathrm{H}_{14} \mathrm{NaO}_{5}$ $[\mathrm{M}+\mathrm{Na}]^{+}:$261.0733; found: 261.0731. Anal. Calcd for $\mathrm{C}_{12} \mathrm{H}_{14} \mathrm{O}_{5}$ (\%): C 60.50, H 5.92, O 33.58; found: C $60.83, \mathrm{H}$ 6.22. Refractive index: 1.482 ; density: 1.268 .

\section{General preparation procedure for monomers NBE-5 and NBE-6}

A dried 2-necked round bottom was charged with the respective functional alcohol ( 2 equiv.), and $N, N$-dimethylaniline ( 2 equiv.). 5-Norbornene-2-carbonyl chloride (1 equiv.) was diluted with $15 \mathrm{ml}$ of dry chloroform and added over $15 \mathrm{~min}$ to the reaction vessel at $0{ }^{\circ} \mathrm{C}$. The mixture was then stirred at RT for $1 \mathrm{~h}$ and further heated to reflux for another $12 \mathrm{~h}$. The reaction was then quenched by adding $10 \mathrm{ml}$ of $6 \mathrm{~N} \mathrm{H}_{2} \mathrm{SO}_{4}$. Afterward, the organic phase was separated and the aqueous phase extracted thrice 
with DCM. The collected organic phases were combined, washed with $6 \mathrm{ml}$ of $6 \mathrm{~N} \mathrm{H}_{2} \mathrm{SO}_{4}$, followed by further washing with $2 \times 20 \mathrm{ml}$ deionized water, $2 \times 20 \mathrm{ml}$ conc. $\mathrm{NaHCO}_{3}$ and $20 \mathrm{ml}$ of brine solution. The crude product in the solution was dried over anhydrous $\mathrm{Na}_{2} \mathrm{SO}_{4}$ and then vacuum evaporated. Finally, the product was purified by column chromatography eluting with heptane and ethyl acetate $(3: 2)$. (Fig. S35-S44, ESI $\dagger$ ).

NBE-5 was obtained as a colourless liquid ( $81 \%$ yield): ${ }^{1} \mathrm{H}$ NMR $\delta: 6.21$ (ddd, $\left.J=22.6,5.7,3.0 \mathrm{~Hz}, 1 \mathrm{H}\right), 6.03$ (ddd, $J=$ $66.8,5.7,3.0 \mathrm{~Hz}, 1 \mathrm{H}), 4.60-4.43(\mathrm{~m}, 2 \mathrm{H}), 3.39-3.28(\mathrm{~m}, 2 \mathrm{H}), 3.22$ $(\mathrm{dq}, J=3.6,2.0 \mathrm{~Hz}, 1 \mathrm{H}), 3.09-2.98(\mathrm{~m}, 1 \mathrm{H}), 3.02(\mathrm{~s}, 3 \mathrm{H}), 2.98-$ $2.93(\mathrm{~m}, 1 \mathrm{H}), 2.01-1.88(\mathrm{~m}, 1 \mathrm{H}), 1.56-1.36(\mathrm{~m}, 2 \mathrm{H}), 1.34-1.25$ (m, 1H). ${ }^{13} \mathrm{C}$ NMR $\delta 175.57,174.02,138.29,138.24,135.51$, 132.01, 57.83, 57.70, 54.04, 49.72, 46.50, 46.40, 45.77, 43.21, $42.94,42.54,42.30,42.27,41.63,30.50,29.31$. MS (ESI) $\mathrm{m} / \mathrm{z}$ calcd For $\mathrm{C}_{11} \mathrm{H}_{16} \mathrm{NaO}_{4} \mathrm{~S}[\mathrm{M}+\mathrm{Na}]^{+}$: 267.0662; found: 267.0662 . Refractive index: 1.475 ; density: 1.267 .

NBE-6 was obtained as a colourless liquid (89\% yield): ${ }^{1} \mathrm{H}$ NMR $\delta 6.20$ (ddd, $J=23.0,5.7,3.0 \mathrm{~Hz}, 1 \mathrm{H}$ ), 6.06 (ddd, $J=$ $60.4,5.7,2.9 \mathrm{~Hz}, 1 \mathrm{H}), 4.35-4.18(\mathrm{~m}, 2 \mathrm{H}), 3.27(\mathrm{dq}, J=3.8,2.1 \mathrm{~Hz}$, $1 \mathrm{H}), 3.11-2.98(\mathrm{~m}, 1 \mathrm{H}), 2.98-2.91(\mathrm{~m}, 1 \mathrm{H}), 2.72(\mathrm{dt}, J=16.1$, $6.3 \mathrm{~Hz}, 2 \mathrm{H}), 2.00-1.90(\mathrm{~m}, 1 \mathrm{H}), 1.55-1.38(\mathrm{~m}, 2 \mathrm{H}), 1.31(\mathrm{dt}, J=$ 8.2, $1.6 \mathrm{~Hz}, 1 \mathrm{H}) .{ }^{13} \mathrm{C}$ NMR $\delta: 175.77,174.25,138.20,138.11$, 135.61, 132.18, 116.89, 116.86, 58.59, 58.47, 49.72, 46.71, 46.36, $45.79,43.17,42.95,42.55,41.67,30.41,29.24,18.10,18.08$. MS (ESI) $\mathrm{m} / z$ calcd for $\mathrm{C}_{11} \mathrm{H}_{13} \mathrm{NNaO}_{2}[\mathrm{M}+\mathrm{Na}]^{+}: 214.0838$; found: 214.0839. Refractive index: 1.482 ; density: 1.133 .

\section{General procedure for ring-opening metathesis polymerization (ROMP)}

Polymers were prepared via ROMP using Grubbs 1st or 3rd generation catalysts, as shown in Scheme 1 below. A 2-necked round bottom flask was charged with the respective monomer NBE-X and DCM. For quantities, see Table 1. The system was initially degassed by two freeze-vacuum-thaw cycles followed by the addition of the catalyst while frozen and a third degassing. The reaction was run under argon and at $40{ }^{\circ} \mathrm{C}$ for $18 \mathrm{~h}$. After confirming total monomer conversion by TLC, ethyl vinyl ether was added, and the resulting mixture stirred for a further $1 \mathrm{~h}$. The product mixture was concentrated under vacuum and then precipitated into excess methanol. The polymer was further purified by five consecutive dissolution (DCM) and re-precipitation cycles $(\mathrm{MeOH})$. The obtained polymers were dried to constant weight under vacuum at $50{ }^{\circ} \mathrm{C}$.

PNBE-2 was obtained as a green solid (98\% yield). ${ }^{1} \mathrm{H}$ NMR (DMSO) $\delta: 7.94(\mathrm{br} \mathrm{s}, 2 \mathrm{H}), 6.71(\mathrm{br} \mathrm{s}, 2 \mathrm{H}), 5.01(\mathrm{br} \mathrm{d}, J=45.8 \mathrm{~Hz}$, $2 \mathrm{H}), 4.09$ (br d, $J=68.8 \mathrm{~Hz}, 2 \mathrm{H}), 3.66$ (br s, $2 \mathrm{H}), 2.99$ (br s, $3 \mathrm{H})$, 2.93-2.16 (br m, 3H), 2.07-0.55 (br m, 4H). ${ }^{13} \mathrm{C}$ NMR (DMSO) $\delta$ : 175.03, 174.00, 154.02, 136.30, 134.54, 133.51, 132.71, 130.76, 129.51, 126.09, 111.07, 61.23, 50.41, 48.10, 44.95, 42.27, 38.88, 37.49, 36.38. (Fig. S45-S47, ESI $\dagger$ ).

PNBE-3 was obtained as a dark red solid (95\% yield). ${ }^{1} \mathrm{H}$ NMR $\delta: 8.26$ (br d, $J=9.0 \mathrm{~Hz}, 2 \mathrm{H}), 7.87(\mathrm{br} \mathrm{s}, 4 \mathrm{H}), 6.78$ (br d, $J=$ $12.2 \mathrm{~Hz}, 2 \mathrm{H}$ ), 5.28 (br dd, $J=44.0,21.3 \mathrm{~Hz}, 2 \mathrm{H}), 4.45-3.87$ (br m, $2 \mathrm{H}$ ), 3.56 (br d, $J=55.7 \mathrm{~Hz}, 4 \mathrm{H}), 3.26-2.61$ (br m, 3H), 1.85 (br d, $J=86.3 \mathrm{~Hz}, 4 \mathrm{H}), 1.46-1.10$ (br m, $3 \mathrm{H}) .{ }^{13} \mathrm{C}$ NMR $\delta: 174.28$,
Table 1 Polymerization of norbornene monomers: Monomer to catalyst ratio [M]:[Cat.], the catalyst used, polymerization degree (n), number average molecular weight $M_{n}$, polydispersity index (PDI), and yield

\begin{tabular}{lllllll}
\hline & & & & & & Yield \\
ID & {$[\mathrm{M}]:[\mathrm{Cat}]$} & Cat. & $n$ & $M_{\mathrm{n}}(\mathrm{Da})$ & PDI & $(\%)$ \\
\hline PNBE-2 & $75: 1$ & Ru-I & 99 & $31300^{a}$ & 1.29 & 92 \\
PNBE-2 & $150: 1$ & Ru-I & 194 & $61300^{a}$ & 1.36 & 95 \\
PNBE-2 & $200: 1$ & Ru-III & 198 & $62800^{a}$ & 2.40 & 94 \\
PNBE-2 & $300: 1$ & Ru-I & 565 & $178700^{a}$ & 1.51 & 96 \\
PNBE-2 & $400: 1$ & Ru-III & 403 & $127600^{a}$ & 2.40 & 97 \\
PNBE-2 & $800: 1$ & Ru-III & 1161 & $367400^{a}$ & 1.59 & 96 \\
PNBE-3 & $150: 1$ & Ru-I & 117 & $50700^{b}$ & 1.46 & 90 \\
PNBE-3 & $200: 1$ & Ru-III & 120 & $52200^{b}$ & 1.33 & 95 \\
PNBE-3 & $300: 1$ & Ru-I & 134 & $58300^{b}$ & 1.87 & 93 \\
PNBE-3 & $400: 1$ & Ru-III & 205 & $88900^{b}$ & 1.46 & 97 \\
PNBE-3 & $800: 1$ & Ru-III & 490 & $213000^{b}$ & 1.56 & 98 \\
PNBE-4 & $75: 1$ & Ru-I & 131 & $31100^{c}$ & 1.60 & 93 \\
PNBE-4 & $150: 1$ & Ru-I & 146 & $34900^{c}$ & 1.91 & 92 \\
PNBE-4 & $200: 1$ & Ru-III & - & - & - & 94 \\
PNBE-4 & $300: 1$ & Ru-I & 136 & $32400^{c}$ & 1.81 & 93 \\
PNBE-4 & $400: 1$ & Ru-III & - & - & - & 95 \\
PNBE-4 & $800: 1$ & Ru-III & - & - & - & 95 \\
PNBE-5 & $200: 1$ & Ru-III & 177 & $43200^{a}$ & 2.48 & 96 \\
PNBE-5 & $400: 1$ & Ru-III & 302 & $73800^{a}$ & 2.41 & 95 \\
PNBE-5 & $800: 1$ & Ru-III & 1956 & $257900^{a}$ & 1.54 & 97 \\
PNBE-6 & $200: 1$ & Ru-III & 187 & $35700^{b}$ & 1.69 & 97 \\
PNBE-6 & $400: 1$ & Ru-III & 276 & $52800^{b}$ & 1.88 & 96 \\
PNBE-6 & $800: 1$ & Ru-III & 470 & $89900^{b}$ & 2.03 & 97
\end{tabular}

${ }^{a}$ GPC in $20 \mathrm{mM}$ sodium trifluoroacetate in HFIP. ${ }^{b}$ GPC in THF. ${ }^{c}$ GPC in HFIP as eluents. The broad PDI observed for same samples may be due to the difficulties faced in solubilizing some samples in the elution solvent or due to chain transfer reactions.

$156.47,154.00,151.31,147.31,143.67,134.52,133.31,132.48$, $130.71,129.80,126.40,124.64,122.57,111.50,60.93,50.12$, $48.71,45.61,42.71,40.51,37.70,36.10,32.77,31.11,26.18$, 25.45, 24.83, 12.36. (Fig. S48-S50, ESI $\dagger$ ).

PNBE-4 was obtained as an off-white solid (98\% yield). ${ }^{1} \mathrm{H}$ NMR (DMSO) $\delta$ : 5.60-4.88 (br m, 3H), 4.58 (br q, $J=7.8 \mathrm{~Hz}, 1 \mathrm{H}$ ), 4.454.00 (br m, 3H), 3.01 (br t, $J=65.8 \mathrm{~Hz}, 3 \mathrm{H}$ ), 2.19-1.06 (br m, 4H). ${ }^{13} \mathrm{C}$ NMR (DMSO) $\delta: 174.86,173.93,155.11,135.27,134.91,133.75$, 132.89, 132.04, 130.98, 129.76, 129.30, 74.77, 74.69, 74.61, 66.51, 66.46, 63.76, 63.66, 49.96, 48.32, 48.08, 45.35, 42.28, 40.89, 40.75, 37.50, 36.48, 35.94, 35.53. (Fig. S51-S53, ESI $\dagger$ ).

PNBE-5 was obtained as an off-white solid (96\% yield). ${ }^{1} \mathrm{H}$ NMR (DMSO) $\delta$ : 5.58-5.06 (br m, 2H), 4.51-4.12 (br m, 2H), 3.48 (br m, $J=8.9,7.9 \mathrm{~Hz}, 2 \mathrm{H}$ ), 3.02 (br s, $J=5.2 \mathrm{~Hz}, 3 \mathrm{H}$ ), 3.28-2.53 (br m, 3H), 2.13-1.19 (br m, 4H). ${ }^{13} \mathrm{C}$ NMR (DMSO) $\delta: 174.90$, 173.89, 135.18, 131.03, 129.88, 58.18, 57.87, 53.20, 53.12, 47.96, 42.22, 42.14, 42.07, 37.48, 36.47, 35.90. (Fig. S54-S56, ESI $\dagger$ ).

PNBE-6 was obtained as an off-white solid (95\% yield). ${ }^{1} \mathrm{H}$ NMR (DMSO) $\delta$ : 5.59-5.03 (br m, 2H), 4.17 (br dddd, $J=$ 32.8, 15.8, 11.1, 5.4 Hz, 2H), 3.29-2.54 (br m, 5H), 2.14-1.33 (br $\mathrm{m}, 4 \mathrm{H}) .{ }^{13} \mathrm{C}$ NMR (DMSO) $\delta: 174.99,173.94,133.80,132.87$, 130.99, 129.75, 118.94, 59.39, 59.24, 59.19, 47.99, 45.39, 42.41, 37.48, 36.51, 35.96, 17.96, 17.88. (Fig. S57-S59, ESI $\dagger$ ).

\section{Results and discussion}

The synthesis of different polar norbornene monomers (NBE-X) is outlined in Scheme 1. Monomers NBE-1 to NBE-4 were 
prepared by an esterification reaction with 5-norbornene-2carboxylic acid, a mixture of endo and exo, whereas monomers NBE-5 and NBE-6 were obtained by esterification reaction of 5-norbornene-2-carboxylic acid chloride, respectively. Monomers were purified by column chromatography over silica gel to achieve a mixture of $80 \%$ endo and $20 \%$ exo-products according to the integration of the olefinic protons in the ${ }^{1} \mathrm{H}$ NMR spectra. The structure of all monomers was confirmed by ${ }^{1} \mathrm{H}$ and ${ }^{13} \mathrm{C}$ NMR spectroscopy, MS spectrometry, and elemental analysis (Fig. S1-S44, ESI $\dagger$ ).

Homopolymerization of NBE-X was carried out with Grubbs I and III catalysts in DCM at $40{ }^{\circ} \mathrm{C}$ for $18 \mathrm{~h}$. Ethyl vinyl ether was used as a quencher. The polymers were isolated in yields of over $90 \%$. Clear evidence for polymerization is provided by ${ }^{1} \mathrm{H}$ NMR spectroscopy images of the vinylene groups. While those of the monomers appear at $\delta=5.8-6.5 \mathrm{ppm}$ those of the polymer absorb at $\delta=5.4 \mathrm{ppm}$. Additionally, all signals of the polymers are broadened. Fig. 1 shows representative ${ }^{1} \mathrm{H}$ NMR spectra of monomer NBE-3 and of polymer PNBE-3. Because the molecular weights of the prepared polymers were relatively high, end groups could not be detected in the ${ }^{1} \mathrm{H}$ NMR spectra. It must be stated that the polymerization of the NBE-1 monomer was unsuccessful. A possible reason might be the stabilization of

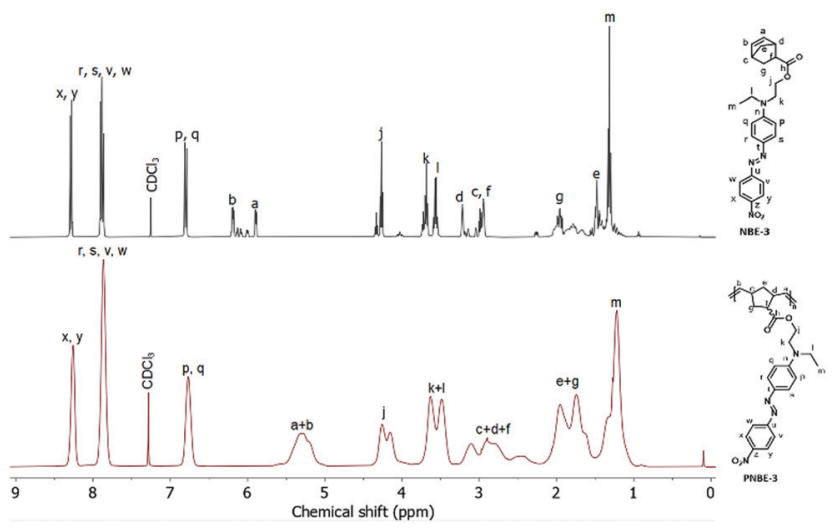

Fig. $1{ }^{1} \mathrm{H}$ NMR spectra in $\mathrm{CDCl}_{3}$ of monomer NBE-3 (top) and its corresponding polymer PNBE-3 (bottom). the reactive metal-carbene complex center by this monomer, thereby inhibiting the polymerization propagation step.

The polymerizations were conducted using varying monomer/catalyst ratios (Table 1$)$. The molecular weights $\left(M_{\mathrm{n}}\right)$ and polydispersities (PDI) were determined by GPC in THF, HFIP, or $20 \mathrm{mM}$ sodium trifluoroacetate solution in HFIP using polystyrene and poly(methyl methacrylate) standards (Table 1). Fig. 2a shows the GPC elugrams of PNBE-3 of different molecular weights. For all other elugrams see the ESI. $\dagger$ The molecular weights of the polymers increased with increasing monomer/ catalyst ratio. The molecular weights calculated based on the ratio of monomer to catalyst are generally higher than those measured by GPC, which is expected as the GPC is a relative method and the standards used for calibration have a different chemical structure. Some polymers show bimodality, presumably due to chain transfer reactions in the later stage of the polymerization.

The thermal behavior of PNBE-X was investigated by differential scanning calorimetry (DSC) and thermogravimetric analysis (TGA) (Fig. 2b and c, Fig. S60-S69, ESI $\dagger$ ). All polymers are amorphous and show a $T_{\mathrm{g}}$. Fig. $2 \mathrm{~b}$ shows the DSC curves of polymers PNBE-X. The corresponding $T_{\mathrm{g}}$ and $\Delta C_{\mathrm{p}}$ values of the transitions are listed in Table 2. The lowest $T_{\mathrm{g}}$ of $62{ }^{\circ} \mathrm{C}$ was measured for PNBE-6 that carries nitrile groups, while the highest $T_{\mathrm{g}}$ of $106{ }^{\circ} \mathrm{C}$ was measured for PNBE-3, which has $N$-ethyl- $N$-(2-hydroxyethyl)-4-(4-nitrophenylazo)aniline (disperse red 1) as a dipole. TGA was carried out to test the thermal stability and decomposition behavior. Polymers PNBE-2, PNBE-4, and PNBE-5 turned out to be stable up to a temperature of $290{ }^{\circ} \mathrm{C}$ (Fig. 2c), while polymers PNBE-6 and PNBE-3 decompose above $200{ }^{\circ} \mathrm{C}$. The molecular weight of the polymers has a negligible impact on thermal behavior, which indicates that the synthesized polymers are in the polymeric regime, where the $T_{\mathrm{g}}$ is molecular weight independent.

Broadband dielectric spectroscopy (DBS) was applied at temperatures between $-100{ }^{\circ} \mathrm{C}$ and $140{ }^{\circ} \mathrm{C}$ to identify the type of mobile segments in the polymers and elucidate the dynamic changes. The measurements were conducted in capacitor geometry, where $100 \mu \mathrm{m}$ thick films of PNBE-X were placed between two metal electrodes with a diameter of $20 \mathrm{~mm}$. The PNBE-X polymers show several relaxation processes within the
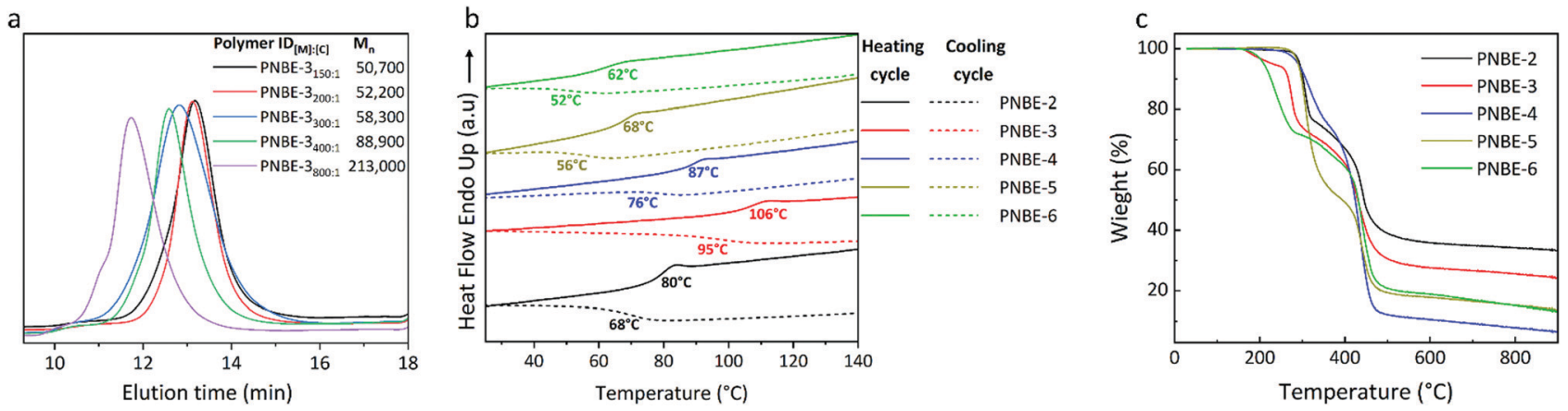

Fig. 2 Molecular weight and thermal characterization; (a) GPC elugrams of PNBE-3 polymers using different monomer/catalyst ratios; (b) DSC curves and (c) TGA thermograms of PNBE-X. 
Table $2 T_{\mathrm{g}}$ values and $\Delta C_{\mathrm{p}}$ of the glass transition, decomposition temperature $T_{\mathrm{d}, 5 \%}$, activation energy $E_{\mathrm{a}}$, room temperature dielectric permittivity, and dielectric relaxation strength $\Delta \varepsilon$

\begin{tabular}{llllllll}
\hline Sample & $M_{\mathrm{n}}(\mathrm{Da})$ & $T_{\mathrm{g}}{ }^{a}\left[{ }^{\circ} \mathrm{C}\right]$ & $T_{\mathrm{g}}{ }^{d}\left[{ }^{\circ} \mathrm{C}\right]$ & $\Delta C_{\mathrm{p}}\left[\mathrm{J} \mathrm{g}^{-1}{ }^{\circ} \mathrm{C}^{-1}\right]$ & $T_{\mathrm{d}, 5 \%}\left[{ }^{\circ} \mathrm{C}\right]$ & $E_{\mathrm{a}}\left[\mathrm{kJ} \mathrm{mol}^{-1}\right]$ & $\varepsilon^{\prime e}$ \\
\hline PNBE-2 & 178700 & $80^{b} / 68^{c}$ & 73 & 0.34 & 294 & $44.15 \pm 0.36$ & 3.37 \\
PNBE-3 & 213000 & $106^{b} / 95^{c}$ & 77 & 0.34 & 222 & $49.66 \pm 0.35$ & 3.84 \\
PNBE-4 & 32400 & $87^{b} / 76^{c}$ & 76 & 0.31 & 289 & $36.08 \pm 0.64$ & 4.97 \\
PNBE-5 & 73800 & $68^{b} / 56^{c}$ & - & 0.23 & 294 & 46.06 \\
PNBE-6 & 52800 & $62^{b} / 52^{c}$ & - & 0.25 & 254 & 38.08 \\
\end{tabular}

${ }^{a} T_{\mathrm{g}}$ taken from DSC measurements. ${ }^{b} T_{\mathrm{g}}$ f for the 2 nd heating and. ${ }^{c} 1$ st cooling profile in the DSC. ${ }^{d}$ Estimated by impedance spectroscopy (IS). ${ }^{e}$ Taken at $25{ }^{\circ} \mathrm{C} .{ }^{f}$ Taken at maximum relaxation.

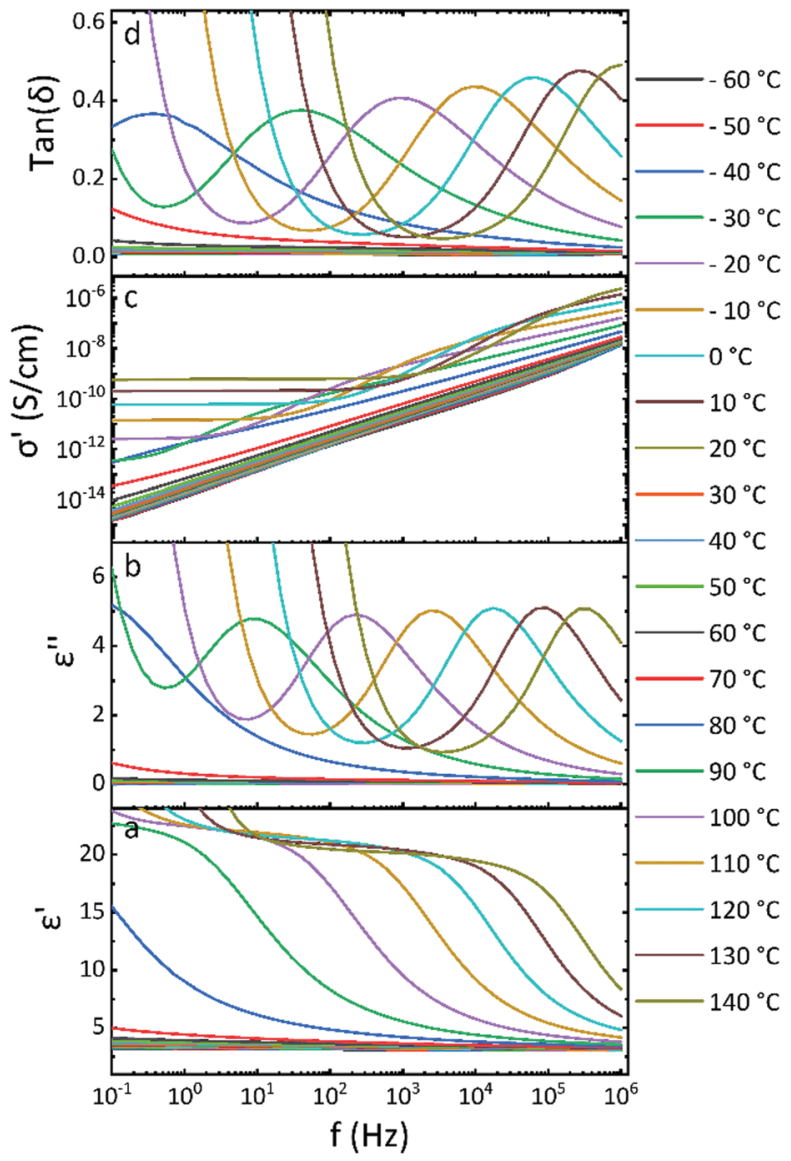

Fig. 3 Isothermal dielectric response of PNBE-2 as a function of frequency; (a) real permittivity $\varepsilon^{\prime}$, (b) dielectric loss $\varepsilon^{\prime \prime}$, (c) real conductivity $\sigma^{\prime}$ and (d) loss $\tan \delta$.

set temperature range. Fig. 3 gives an illustration of how the real part $\varepsilon^{\prime}$ and imaginary part $\varepsilon^{\prime \prime}$ of the complex permittivity, the real part of the conductivity $\sigma^{\prime}$ and the loss tangent $\tan \delta$ evolve with the temperature at different frequencies for PNBE-2. Generally, there is a noticeable increase in $\varepsilon^{\prime}$ with increasing temperature over the specified frequency range, as shown in Fig. 3a. However, the $\varepsilon^{\prime}$ remains virtually constant from $-60{ }^{\circ} \mathrm{C}$ to $70{ }^{\circ} \mathrm{C}$ because the orientation motions are restricted to small angles. Above $70{ }^{\circ} \mathrm{C}$ the increment becomes very pronounced, which is attributed to the thermal activation of the dipole motions in the polymer. Thus, the presence of pendant dipolar moieties on the polymer chains experiences enough hindrances, which impose potential energy barriers to their mobility below certain temperatures. Such energy impositions are dependent on the packing density, the intrinsic chain flexibility, rotational restrictions, softness of potential related to angle changes, and the intra- and intermolecular dipole interactions. $^{31,33}$

Adding enough thermal energy to the system helps overcome these energy barriers, and segmental rotation allows reorientation of the dipoles reflected in an increased polarization. Thus, the orientation polarization of the dipoles occurs above $T_{\mathrm{g}}$. The relaxation peak for the orientation polarization process is shifted to higher frequencies with increasing temperature. Above $100{ }^{\circ} \mathrm{C}$, the ionic conductivity contributes to the increase in dielectric permittivity at low frequencies. This is also supported by the dielectric loss, which shows an increase at low frequencies with a slope approximation of -1 and a sudden increase in the conductivity of the material, as shown in Fig. 3b-d. The temperature increases the ionic conductivity and shifts this process to higher frequencies.

To relate the bulk dielectric behavior of PNBE-X polymers with respect to structural and molecular motions of their dipolar segments, an approximation was made by fitting the experimental data with a Havriliak-Negami (HN) relaxation function. This was achieved through fitting isothermal data of $\varepsilon^{\prime \prime}$ by a superposition of the HN-function and a conductivity contribution as shown in eqn (2) below ${ }^{34}$

$$
\varepsilon^{\prime \prime}=\frac{\sigma_{0}}{\varepsilon_{0}} \frac{a}{\omega^{s}}+\operatorname{Im}\left[\frac{\Delta \varepsilon}{\left(1+(i \omega \tau)^{\alpha}\right)^{\gamma}}\right]
$$

where $\varepsilon^{\prime \prime}$ is the imaginary permittivity, $\sigma_{0}$ is the d.c.conductivity, $a$ is a constant, $\varepsilon_{0}$ the permittivity of vacuum, $\omega=2 f$ the angular frequency, $\Delta \varepsilon$ the dielectric relaxation strength, $\tau$ the relaxation time, $\alpha$ and $\gamma$ describe the symmetric and asymmetric broadening of the relaxation peak, $i$ the imaginary unit $(i=\sqrt{-1})$. The exponent $s<1$ are observed for ionic charge carriers, which cause electrode polarisation. The parameters of relevance to understand the dynamic transitions taken place at the molecular level and the possibility to polarize these polymers are $\tau$ and $\Delta \varepsilon$.

Generally, two distinct regimes revealing molecular motions responsible for $\alpha$ - and $\beta$-relaxation processes in glass-forming materials were easily fitted, as illustrated in Fig. 4a. Complete sets of fits for all polymers can be found in the ESI $\dagger$ (Fig. S70S82). The relaxation times for the $\beta$-processes, as shown in Fig. 4b, exhibit Arrhenius temperature dependences over the 

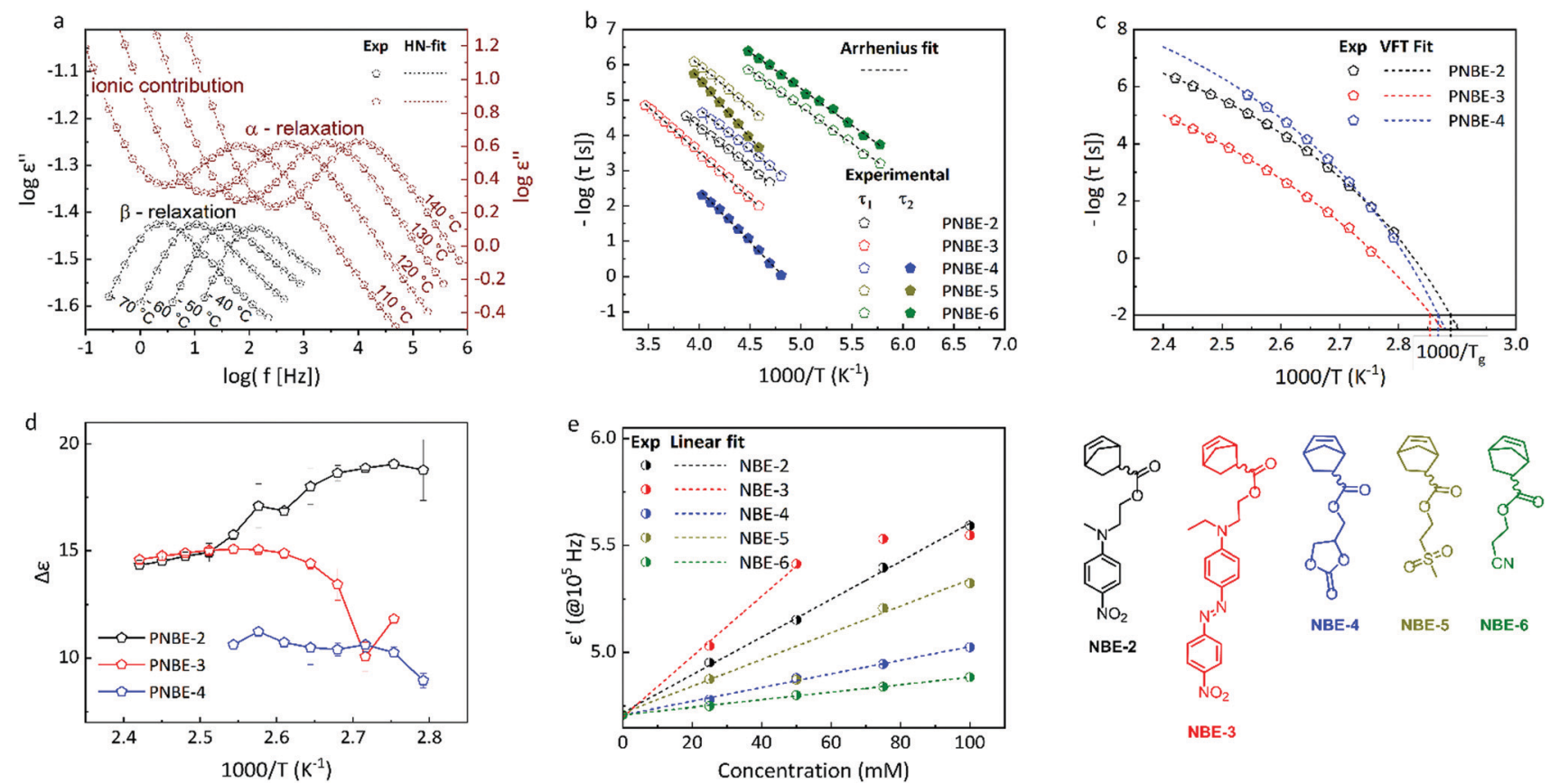

Fig. 4 Dielectric relaxation of PNBE-X polymers and the dipolar dynamics of their respective monomers; (a) isothermal illustration of the imaginary part $\varepsilon^{\prime \prime}$ of the complex dielectric function vs. frequency for two temperature regimes; below $T_{\mathrm{g}}$ ( $\beta$-relaxation) and above $T_{\mathrm{g}}$ ( $\alpha$-relaxation) for PNBE-3; (b) Arrhenius fitting of $\beta$-relaxation mechanisms for PNBE-X; (c) VFT plot of $\alpha$-relaxation processes for PNBE-2, PNBE-3, and PNBE-4; and (d) temperature dependence of dielectric relaxation strength for PNBE-2, PNBE-3, and PNBE-4 in the $\alpha$-relaxation regime; (e) dielectric permittivity as a function of concentration for NBE-X in chloroform. From the slopes the respective dipole moments were calculated.

temperature range in which the process is resolvable. The Arrhenius fits describe the experimentally determined relaxation times. The activation energies, which depend on both internal rotational barriers and the environment of the dipolar fluctuating unit, obtained from the fitting for the PNBE-X polymers are listed in Table 2 . In contrast, the $\alpha$-relaxation processes for PNBE-X polymers exhibit clear non-Arrhenius temperature dependencies, well represented by a VogelFulcher-Tammann (VFT) fit function with parameters in good agreement with experimental values as shown in Fig. 4c. The degree of deviation from an Arrhenius-type temperature dependence provides a useful classification of glass-forming systems. Additionally, at the calorimetric glass transition temperature $T_{\mathrm{g}}$, the $\alpha$-relaxation reaches a peak frequency approximated to $10^{-3} \mathrm{~Hz}^{34}$ The $T_{\mathrm{g}}$ of glass-forming materials can be extrapolated from the $\alpha$-relaxation processes obeying a VFT-temperature dependence. As a result, the $T_{g}$ for PNBE-2, PNBE-3, and PNBE-4 could be estimated by DBS techniques as listed in Table 2. While the $\beta$-processes are believed to be due to localized motions of the pendant dipolar moieties, the $\alpha$-processes are attributed to segmental relaxations of the polymer chains. For amorphous polymers, $\Delta \varepsilon$ is said to decrease with increasing temperature above $T_{\mathrm{g}}{ }^{34}$ Fig. $4 \mathrm{~d}$ shows the temperature dependence of $\Delta \varepsilon$ for PNBE-2, PNBE-3, and PNBE-4 polymers within the $\alpha$-relaxation regime. This temperature dependency was more pronounced in PNBE-2 polymer than in the PNBE-3 and PNBE-4 polymers. The maximum dielectric relaxation strengths were estimated to be 19.06, 15.08, and 11.24 for PNBE-2, PNBE-3, and PNBE-4, respectively. For PNBE-5 and
PNBE-6 we could not assess the relaxation strength because of the high ionic conductivity of the samples.

To clarify the observed trend in dielectric relaxation strengths $\Delta \varepsilon$ of PNBE-X polymers, the molecular dipole moments of the corresponding NBE-X monomers were evaluated experimentally. The $\Delta \varepsilon$ is proportional to the dipole moments of contributing dipolar units within a material. ${ }^{34}$ The dipole moments of NBE-X were determined from dielectric spectroscopy measurements of solutions of NBE-X in chloroform at ambient temperature and $10^{5} \mathrm{~Hz}$. To avoid dipolar interactions, dilute solutions of the monomers in chloroform were prepared. The dielectric permittivity was measured as a function of solute concentration, as shown in Fig. 4e. Dipole moments were thereafter estimated by modified Onsager equation according to Böttcher ${ }^{35,36}$ and a model according to HedestrandGuggenheim-Smith. ${ }^{37,38}$ The obtained results are listed in Table 3. For the equations used, please see ESI. $\dagger$ The dipole

Table 3 Dipole moments of NBE-X determined by Hedestrand-Guggenheim-Smith (HGS) model and Böttcher model

\begin{tabular}{lllrr}
\hline & & & \multicolumn{2}{c}{ Dipole moment (Debye) } \\
\cline { 4 - 5 } Monomer & $\rho\left[\mathrm{g} \mathrm{cm}^{-3}\right]$ & \multicolumn{1}{l}{$n$} & \multicolumn{1}{c}{$\mu_{\text {HGS }}$} & \multicolumn{1}{c}{$\mu_{\text {Böttcher }}$} \\
\hline NBE-1 & 1.238 & 1.565 & $12.21 \pm 2.38$ & $11.54 \pm 1.09$ \\
NBE-2 & 1.237 & 1.582 & $8.67 \pm 1.26$ & $9.34 \pm 1.28$ \\
NBE-3 & 1.185 & 1.422 & $11.54 \pm 1.37$ & $10.90 \pm 1.76$ \\
NBE-4 & 1.268 & 1.482 & $4.52 \pm 0.87$ & $7.67 \pm 1.47$ \\
NBE-5 & 1.267 & 1.475 & $6.41 \pm 1.32$ & $8.63 \pm 1.51$ \\
NBE-6 & 1.133 & 1.482 & $3.00 \pm 0.42$ & $7.07 \pm 1.63$
\end{tabular}


moment was obtained from the slope of $\varepsilon^{\prime}$ versus molar concentration (Fig. 4e). As expected, the highest dipole moment was found for NBE-1, but this monomer did not polymerize (Table 3). The second highest dipole was found for NBE-3. However, this high dipole moment is not reflected in the relaxation strength of PNBE-3, which was lower than for NBE-2, which has a lower dipole moment. The low relaxation strength of PNBE-3 may be related to the different dipole moments of cis-trans isomers of disperse red 1 or the antiparallel orientation of the dipoles, which is likely favored by the $\pi-\pi$ interactions.

\section{Conclusions}

Six polar norbornene monomers were synthesized and five of them were successfully polymerized by ROMP using Grubbs I and III generation catalysts. The molecular weight of the polymers, as revealed by GPC, show increasing correlation with the theoretical monomer-to-catalyst feed ratio. The synthesized polymers show moderate room temperature dielectric permittivity, which is typical for high $T_{\mathrm{g}}$ polar glassy polymers. All polymers show an increase in the dielectric relaxation strength at temperatures above the $T_{\mathrm{g}}$. Because of their high dielectric relaxation strength, we propose the novel polymers to be useful as electrets and to find applications in thermal sensors and thermal energy harvesting. Work in this direction is underway.

\section{Author contributions}

F. O. conducted all experimental work and recorded the impedance spectroscopy data as well as the fittings of the dielectric data. D. M. O. initiated the work, raised the funding, and supervised the research. M. T. supported the interpretation of the dielectric measurements. F. N. supported the calculations of the dipole moment. S. L. supported the GPC measurements. All authors contributed to scientific discussions. F. O. and D. M. O. wrote the manuscript with input from all authors.

\section{Conflicts of interest}

There are no conflicts to declare.

\section{Acknowledgements}

This project has received funding from the Swiss National Science Foundation (IZSAZ2_173358/1 and 206021_150638/1), the Swiss Federal Laboratories for Materials Science and Technology (Empa, Dübendorf, Switzerland), and the European Research Council (ERC) under the European Union's Horizon 2020 research and innovation programme (grant agreement No. 101001182). We acknowledge B. Fischer (Empa) for DSC and TGA measurements, Dr D. Rentsch (Empa) for help with NMR measurements, E. Hack (Empa) for the refractive index measurements and for reading the manuscript, and Y. Lavanchy from EPFL for some GPC measurements. We also acknowledge Prof. A. Schönhals (BAM, Berlin, Germany) for valuable discussions.

\section{References}

1 Q. M. Zhang and M. J. Serpe, ChemPhysChem, 2017, 18, 1451-1465.

2 A. S. Abd-El-Aziz, M. Antonietti, C. Barner-Kowollik, W. H. Binder, A. Böker, C. Boyer, M. R. Buchmeiser, S. Z. D. Cheng, F. D'Agosto, G. Floudas, H. Frey, G. Galli, J. Genzer, L. Hartmann, R. Hoogenboom, T. Ishizone, D. L. Kaplan, M. Leclerc, A. Lendlein, B. Liu, T. E. Long, S. Ludwigs, J.-F. Lutz, K. Matyjaszewski, M. A. R. Meier, K. Müllen, M. Müllner, B. Rieger, T. P. Russell, D. A. Savin, A. D. Schlüter, U. S. Schubert, S. Seiffert, K. Severing, J. B. P. Soares, M. Staffilani, B. S. Sumerlin, Y. Sun, B. Z. Tang, C. Tang, P. Théato, N. Tirelli, O. K. C. Tsui, M. M. Unterlass, P. Vana, B. Voit, S. Vyazovkin, C. Weder, U. Wiesner, W.-Y. Wong, C. Wu, Y. Yagci, J. Yuan and G. Zhang, Macromol. Chem. Phys., 2020, 221, 2000216.

3 S. Bauer, IEEE Trans. Dielectr. Electr. Insul., 2006, 13, 953-962. 4 H. Kawai, Jpn. J. Appl. Phys., 1969, 8, 975-976.

5 Q. Li and Q. Wang, Macromol. Chem. Phys., 2016, 217, 1228-1244.

6 X. Chen, X. Han and Q.-D. Shen, Adv. Electron. Mater., 2017, 3, 1600460.

7 R. Gerhard, From electrode charges on dielectric elastomers to trapped charges and electric dipoles in electrets and ferroelectrets: fundamental and applications-relevant aspects of diversity in electroactive polymers, SPIE, 2016, 9798, 97980T-1.

8 X. Qiu, W. Wirges and R. Gerhard, J. Appl. Phys., 2011, 110, 024108.

9 Y. S. Ko, F. A. Nüesch, D. Damjanovic and D. M. Opris, $A d v$. Mater., 2017, 29, 1603813.

10 H. K. Hall Jr., A. B. Padias, G. Chu, H.-Y. Lee, I. Kalnin, M. Sansone and G. Breckenridge, J. Polym. Sci., Part A: Polym. Chem., 1992, 30, 2341-2347.

11 G. R. Davies, H. V. S. A. Hubbard, I. M. Ward, W. J. Feast, V. C. Gibson, E. Khosravi and E. L. Marshall, Polymer, 1995, 36, 235-243.

12 F. Kremer, L. Dominguez, W. H. Meyer and G. Wegner, Polymer, 1989, 30, 2023-2029.

13 S. Bauer and S. B. Lang, IEEE Trans. Dielectr. Electr. Insul., 1996, 3, 647-676.

14 T. L. Choi and R. H. Grubbs, Angew. Chem., Int. Ed., 2003, 42, 1743-1746.

15 K.-T. Bang and T.-L. Choi, J. Polym. Sci., 2020, 58, 48-51.

16 S. Hilf and A. F. M. Kilbinger, Nat. Chem., 2009, 1, 537-546.

17 A. Leitgeb, J. Wappel and C. Slugovc, Polymer, 2010, 51, 2927-2946.

18 D. Ndaya, R. Bosire and R. M. Kasi, Polym. Chem., 2019, 10, 3868-3878.

19 N. R. Grove, P. A. Kohl, S. A. Bidstrup Allen, S. Jayaraman and R. Shick, J. Polym. Sci., Part B: Polym. Phys., 1999, 37, 3003-3010. 
20 M. A. Rahman, H. N. Lokupitiya, M. S. Ganewatta, L. Yuan, M. Stefik and C. Tang, Macromolecules, 2017, 50, 2069-2077.

21 M. S. Ganewatta, W. Ding, M. A. Rahman, L. Yuan, Z. Wang, N. Hamidi, M. L. Robertson and C. Tang, Macromolecules, 2016, 49, 7155-7164.

22 Y. Shao, C. Lavigueur and X. X. Zhu, Macromolecules, 2012, 45, 1924-1930.

23 Y. Zhu, C. Ma, H. Han, R. Sun, X. Liao and M. Xie, Polym. Chem., 2019, 10, 2447-2455.

24 Z. You, D. Gao, O. Jin, X. He and M. Xie, J. Polym. Sci., Part A: Polym. Chem., 2013, 51, 1292-1301.

25 G. O. Karpov, D. A. Alentiev, A. I. Wozniak, E. V. Bermesheva, I. V. Lounev, Y. A. Gusev, V. P. Shantarovich and M. V. Bermeshev, Polymer, 2020, 203, 122759.

26 L. Fang, J. Zhou, Y. Tao, Y. Wang, X. Chen, X. Chen, J. Hou, J. Sun and Q. Fang, ACS Sustainable Chem. Eng., 2019, 7, 4078-4086.

27 J. Wang, J. Zhou, L. Fang, J. Sun and Q. Fang, Mater. Chem. Front., 2018, 2, 1467-1474.

28 H. Yin, P. Chapala, M. Bermeshev, A. Schönhals and M. Böhning, ACS Macro Lett., 2017, 6, 813-818.
29 H. Han, D. Zhou, Q. Ren, F. Ma, C. Ma and M. Xie, Eur. Polym. J., 2020, 122, 109376.

30 S. Bonardd, A. Alegria, C. Saldias, A. Leiva and G. Kortaberria, ACS Appl. Mater. Interfaces, 2018, 10, 38476-38492.

31 S. Bonardd, Á. Alegria, O. Ramirez, C. Saldías, Á. Leiva and G. Kortaberria, React. Funct. Polym., 2019, 140, 1-13.

32 Y. S. Ko, F. A. Nüesch and D. M. Opris, J. Mater. Chem. C, 2017, 5, 1826-1835.

33 R. H. Boyd and G. D. Smith, Polymer Dynamics and Relaxation, Cambridge University Press, New York, 2007, p. $160,187$.

34 F. Kremer, Broadband dielectric spectroscopy, Springer, Berlin, 2003.

35 C. J. F. Böttcher, Recl. Trav. Chim. Pays-Bas, 1943, 62, 119-133.

36 J. Wudarczyk, G. Papamokos, T. Marszalek, T. Nevolianis, D. Schollmeyer, W. Pisula, G. Floudas, M. Baumgarten and K. Müllen, ACS Appl. Mater. Interfaces, 2017, 9, 20527-20535.

37 J. Knelles, C. Wanner, F. Schulz, M. Freund, M. A. Kolmangadi, A. Baro, P. Huber, A. Schönhals and S. Laschat, Liq. Cryst., 2021, 48, 1382-1391.

38 E. A. Guggenheim, Trans. Faraday Soc., 1951, 47, 573-576. 